\title{
Bending instabilities at the origin of persistent warps: a new constraint on dark matter halos
}

\author{
Y. Revaz* and D. Pfenniger \\ Geneva Observatory, Switzerland \\ E-mail: yves.revaz@obs.unige.ch, daniel.pfenniger@obs.unige.ch
}

\begin{abstract}
Based on N-body simulations, we show that realistic galactic disks are subject to bending instabilities of fire-hose type when the disks are substantially self-gravitating, that is, if they contain dark matter distributed in the disk. Depending on the degree of instability, S and U-shaped, as well as asymmetric warps are generated. In some cases, the warp may last several galactic rotations, particularly when the instability is marginal. Since the bending instability is very sensitive to the disk flattening, the fractions of dark matter distributed in the disk and in the dark halo are constrained. For a Milky Way like galaxy the extended dark halo can not exceed 30-40\% of the total mass within $35 \mathrm{kpc}$ if the Milky Way warp results from a bending instability. This mode of warping provides a unified picture of spiral galaxies, where bars, spiral arms and warps result all from disk gravitational instabilities, radial or transverse, which are constantly regenerated by the dissipative gas component.
\end{abstract}

Baryons in Dark Matter Halos

5-9 October 2004

Novigrad, Croatia

${ }^{*}$ Speaker.

Published by SISSA

http://pos.sissa.it/ 


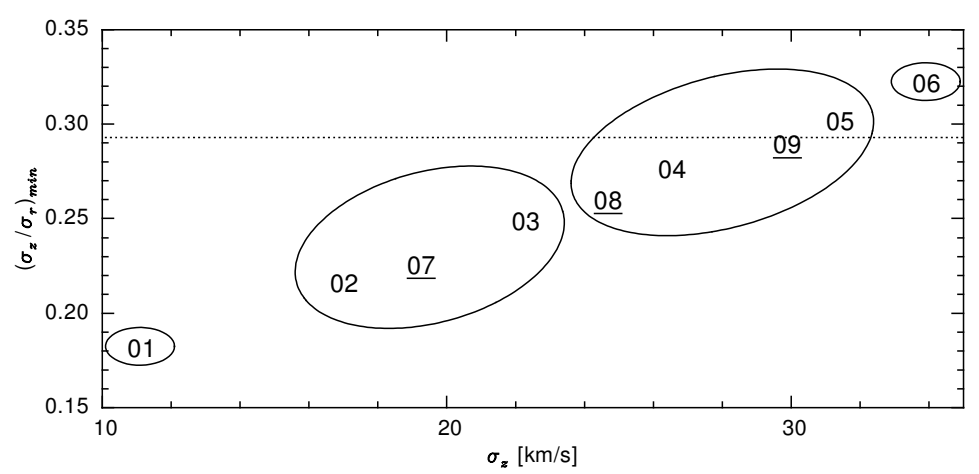

Figure 1: Ratio $\sigma_{z} / \sigma_{R}$ as a function of the vertical dispersion $\sigma_{z}$ at $R=15 \mathrm{kpc}$. The values are taken at the radius where $\sigma_{z} / \sigma_{R}$ is minimum. The dotted line corresponds to Araki's limit.

\section{Introduction}

Since the first observation of the Milky Way warp at the end of the fifties, warped galaxies have represented a challenge for astrophysicists (see for example Binney, 1992, for a review). Several hypotheses like normal modes, accretion events, or dynamical friction have been proposed to explain their ubiquity. Most of the explanations assume that galactic disks are embedded in a dominant spheroidal dark halo. However, there are now several observations (e.g. of NGC 2915) which can be best explained with a substantial amount of dark matter in the outer galactic disks (Masset \& Bureau 2003), in agreement with cold and clumpy gas dark matter models distributed like HI disks (Pfenniger \& Combes, 1994). In this paper, we summarize the recent result presented in Revaz \& Pfenniger (2004) where warps are spontaneously generated by bending instabilities (see for example Fridman \& Polyachenko, 1984, for a formal description of this instability) if a substantial fraction of the dark matter resides in the disk of spiral galaxies.

\section{Initial N-body models}

In order to study the possible spontaneous vertical bending of spiral galaxies, we have first studied the evolution of equilibrium N-body models starting with different thicknesses, and without any dark halo. Each model contains a bulge, an exponential disk and a dark matter heavy disk parametrized by its thickness $h_{z 0}$. The initial vertical velocity dispersion $\sigma_{z}$ is obtained by satisfying the equilibrium solution of the Jeans equation, separately for each component $i$. The radial and azimuthal velocities dispersions $\sigma_{R}$ and $\sigma_{\phi}$ are obtained from $\sigma_{z}$, using the ratio between the epicycles frequencies $\kappa$ and $v$, and the rotation frequency $\Omega$ :

$$
\rho_{i} \sigma_{z i}^{2}=\int_{z}^{\infty} d z \rho_{i} \partial_{z} \Phi, \quad \frac{\sigma_{\phi}^{2}}{\sigma_{R}^{2}}=\frac{\kappa^{2}}{4 \Omega^{2}} \quad \text { and } \quad \frac{\sigma_{z}^{2}}{\sigma_{R}^{2}}=\beta^{2} \frac{\kappa^{2}}{v^{2}} .
$$

The free factor $\beta$ is chosen of order 1 to keep the Savronov-Toomre radial stability parameter $Q$ around 1.5. Fig. 1 shows the Araki ratio $\sigma_{z} / \sigma_{R}$ (Araki, 1985) as a function of the vertical dispersion $\sigma_{z}$. Thin disks lie at lower left end while thick disks at the upper right end. 


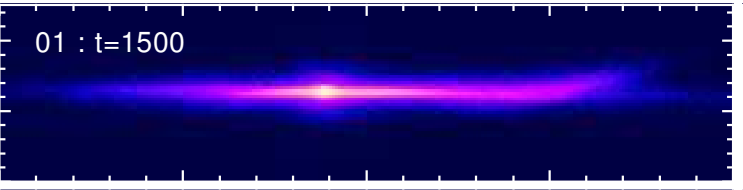

$04: t=3500$
$02: t=2000$

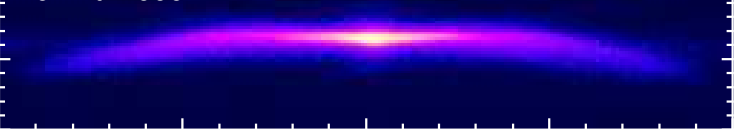

$06: t=2000$
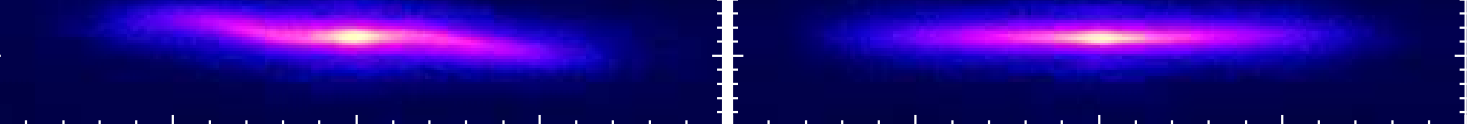

Figure 2: Edge-on projections of the models 01, 02, 04, and 06. Time in Myr is indicated at the upper left. The box dimensions are $100 \times 25 \mathrm{kpc}^{2}$

\section{Simulations and results}

As expected from theory, the stability of the models depends strongly on the precise thickness of the heavy disk. In Fig. 1, from left to right, models can be divided into four groups. A characteristic snapshot of each group is displayed in Fig. 2.

1) Very thin models like model $01\left(h_{z 0}<150 \mathrm{pc}\right)$ have a ratio $\sigma_{z} / \sigma_{R}$ below 0.2 . They are highly unstable and generate an asymmetric warp that extends up to $z=4 \mathrm{kpc}$ at $R=35 \mathrm{kpc}$.

2) Models 02,07 and $03\left(h_{z 0}=150-250 \mathrm{pc}\right)$ have still a ratio $\sigma_{z} / \sigma_{R}$ well below the Araki limit. The bending instability occurs during the first $2 \mathrm{Gyr}$. A spectacular axisymmetric bowl mode $(m=0)$ grows during about $1 \mathrm{Gyr}$, before that $\sigma_{z}$ increases, which stabilizes the disk.

3) In the models $08,04,09$ and $05\left(h_{z 0}=250-450 \mathrm{pc}\right)$, the ratio $\sigma_{z} / \sigma_{R}$ is almost critical with respect to Araki's limit. These models develop S-shaped warped modes $(m=1)$. In the case of model 08, the warp is long-lived and lasts more than $5.5 \mathrm{Gyr}$.

4) The thickest model, $06\left(h_{z 0} \geq 550 \mathrm{pc}\right)$ has a ratio $\sigma_{z} / \sigma_{R}$ well above 0.3 . The disk remains stable.

\section{The influence of an extended dark halo}

The previous models have been run without any outer dark halo, showing that the initial $\sigma_{z} / \sigma_{R}$ ratio in the initial N-body runs does determine the warp growth. The influence of an external dark halo on Araki's limit is then studied semi-analytically by embedding the previous models in a Miyamoto-Nagai potential. The heavy disk to total dark mass ratio $f$ and the halo flattening $\zeta_{h}$ (iso-density axis ratio) characterize the semi-analytical models. For a realistic fixed disk thickness $h_{z 0}=250 \mathrm{pc}$, Fig. 3 shows the ratio $\sigma_{z} / \sigma_{R}$ at its minimum value along the radius, indicating the stability of the disk. In the absence of the halo $(f=1)$, the model is unstable. At the opposite, when the heavy disk is replaced by a spherical halo $\left(\zeta_{h}=1\right)$ of equal mass $(f=0)$, the disk is stabilized, as long as the halo flattening is realistic $\left(\zeta_{h}>0.5\right)$. For very small value of $\zeta_{h}$, the halo plays the role of the disk and the model becomes unstable. For a realistic $\zeta$, the halo flattening has little effect on the stability. In contrast the dark matter fraction in the halo is well constrained: the disk remains stable as long as the heavy disk to dark matter mass ratio is smaller than 0.5 , in other words, bending instabilities appear only if the dark disk is heavier than the dark halo. 


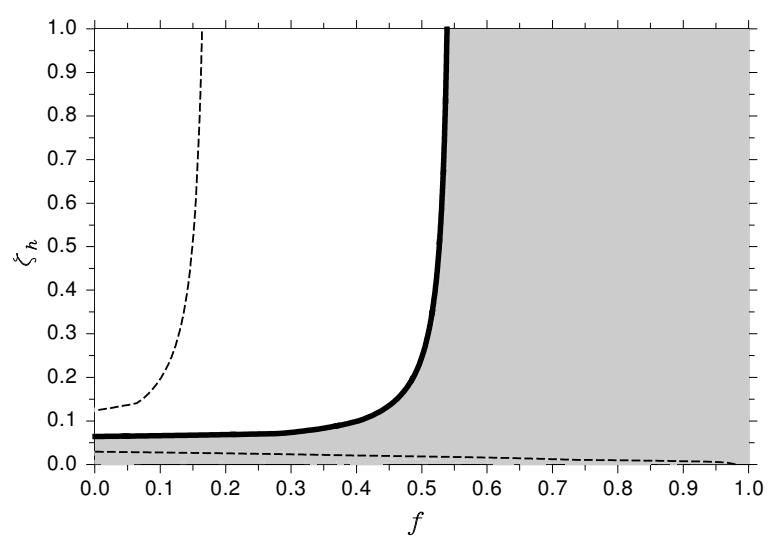

Figure 3: Stability of a realistic model $h_{z 0}=250 \mathrm{pc}$ against bending oscillations. The radial minimum value of $\sigma_{R} / \sigma_{z}$ is plotted as a function of the dark matter fraction $f$ in the heavy disk and of the halo flattening $\zeta_{h}$. The solid line corresponds to the Araki critical value of 0.293, the upper dashed line to the values of 0.4 , the bottom dashed line to 0.2 . The shaded region corresponds to unstable models.

\section{Conclusions}

Our numerical N-body simulations have shown that heavy disks may be subject to bending instabilities yielding $\mathrm{S}, \mathrm{U}$ or asymmetric warps. The shape and amplitude of these warps are similar to the observed optical warps. This scenario gives also an interesting constraint on the halo mass in spiral galaxies. For realistic disk thickness, bending instabilities may appear only if the halo is less massive than about $0.3-0.4$ times the total galactic mass within the warped disk radius.

As discussed in more detail in Pfenniger \& Revaz (2004), the dissipational behavior of the gas (not taken into account in the present simulations) reduces the gas velocity perpendicular to the galactic plane while, in the plane, higher values of velocities are maintained by the dynamical heating due to instabilities like bars and spirals. As a consequence, we expect that a galactic disk naturally maintains a regime marginally unstable with respect to bending instabilities. Like spiral arms, warps can be repeatedly excited as long as the dissipative component, the gas, exists.

\section{References}

Araki, S., 1985, Ph.D. Thesis, Massachusetts Inst. Technology

Binney, J. 1992, ARA\&A, 30, 51

Fridman, A.M., Polyachenko, V.L., 1984 Physics of Gravitating Systems, New York: Springer

Masset, F. S. \& Bureau, M. 2003, ApJ, 586, 152

Pfenniger, D., Revaz, Y. 2004, in "Penetrating Bars through Masks of Cosmic Dust: The Hubble

Tuning Fork Strikes a New Note”, D. Block et al. (eds.), Kluwer, in press (astro-ph/0406578)

Pfenniger, D. \& Combes, F. 1994, A\&A, 285, 94

Revaz, Y., Pfenniger, D. 2004, A\&A, 425, 67 